\title{
Management of pediatric uveitis
}

\section{Bailey A. Wentworth ${ }^{1,2}$, Clovis A. Freitas-Neto ${ }^{1,2}$ and C. Stephen Foster ${ }^{1,2,3 *}$}

\author{
Addresses: ${ }^{1}$ Massachusetts Eye Research and Surgery Institution (MERSI), 5 Cambridge Center, Cambridge, MA 02142, USA; ${ }^{2}$ Ocular \\ Immunology and Uveitis Foundation, 348 Glen Road, Weston, MA 02493, USA; ${ }^{3}$ Department of Ophthalmology, Harvard Medical School, \\ 25 Shattuck Street, Boston, MA 02115, USA \\ * Corresponding author: C. Stephen Foster (sfoster@mersi.com) \\ FI000Prime Reports 2014, 6:4I (doi:10.12703/P6-4I) \\ All Fl000Prime Reports articles are distributed under the terms of the Creative Commons Attribution-Non Commercial License \\ (http://creativecommons.org/licenses/by-nc/3.0/legalcode), which permits non-commercial use, distribution, and reproduction in any medium, \\ provided the original work is properly cited.
}

The electronic version of this article is the complete one and can be found at: http://fl000.com/prime/reports/m/6/4 I

\begin{abstract}
Pediatric uveitis is a topic of special interest not only because of the unique diagnostic and therapeutic challenges but also because of the lifetime burden of vision loss if the problem is not adequately treated, as well as the economic and psychological toll on the family. Often, uveitis in children is discovered as part of a routine eye exam; this silent, insidious inflammation can be difficult to treat and can lead to further complications if not handled skillfully. Corticosteroids have long been the mainstay of therapy; however, the significant associated side effects mandate a corticosteroid-sparing therapeutic regimen in pursuit of remission. In this review, we cover the therapeutic options for pediatric uveitis, specifically focusing on the most common non-infectious varieties, juvenile idiopathic arthritis-associated uveitis and pars planitis.
\end{abstract}

\section{Introduction}

While the incidence and prevalence of uveitis in children is less than that of adults $[1,2]$, pediatric uveitis deserves special attention because of its unique diagnostic and therapeutic challenges, the lifetime burden of blindness if the problem is not cured, and the economic and psychological toll on the family. Children make up between $2.2 \%$ and $13.8 \%$ of patients in uveitis clinics [1-3]. Uveitis in children is often discovered as part of a routine eye exam, as many children do not report any symptoms, and is more likely to be chronic, recurrent, and difficult to treat. Providers may also face difficulty in getting a complete history and review of systems in uncooperative or preverbal children; general anesthesia is sometimes necessary for a complete exam in very young children.

The risk of poor visual outcomes in children may be greater than in the adult population, due to a delay in diagnosis and established ocular pathology. Between suboptimal therapy and tolerance to insidious inflammation, children are prone to complications and may silently develop cataract, glaucoma, band keratopathy, or amblyopia. Furthermore, the presence of complications or established pathology at presentation is a significant risk factor for further complications $[4,5]$.

Treatment options are many, and providers must be conscious of the potentially vision-threatening prognosis of uveitis in a child who is not treated quickly and appropriately. In the cases that are not resolved by initial corticosteroid therapy, providers must be keen to initiate immunomodulatory therapy early on. Extensive use of corticosteroids in children can have significant side effects; the child with recalcitrant uveitis should be co-managed with a rheumatologist or referred immediately to an ophthalmologist with the ability to adequately establish and monitor immunosuppressive therapies, such as classic immunomodulators or biologic response modifiers.

\section{Differential diagnosis}

Uveitis in children can generally be identified as having one of three following causes: infectious; non-infectious (e.g. as part of an underlying autoimmune syndrome); or as a manifestation of a masquerade syndrome. The clinical investigation should be initiated and conducted based on a detailed family and past medical history, the 
age of the child (Table 1), anatomical location, and granulomatous or non-granulomatous inflammation (Table 2) [6]. The variety of manifestations in numerous uveitic entities is broad and includes many of the same entities seen in adults. Infectious and non-infectious entities of special importance to the pediatric age group are summarized in this article by the most common causes.

\section{Infectious vs. non-infectious uveitis}

There is an estimated incidence of 4.9-6.9 per 100,000 person-years, and prevalence of $13-30$ per 100,000 population, of non-infectious anterior uveitis in the North American population [7]. Juvenile idiopathic arthritis is the most common systemic association of pediatric uveitis [7].

Treatment for non-infectious pediatric uveitis is multifactorial. Topical and oral corticosteroids are used to treat acute inflammation. Corticosteroid-sparing agents should be introduced to control the inflammation. Immunomodulatory therapy has been associated with positive outcomes in non-infectious pediatric uveitis, but therapy can be limited by medication-related side effects [8].

Infectious diseases such as toxoplasmosis, toxocariasis, Lyme disease, syphilis, tuberculosis, and some viruses can be common causes of pediatric uveitis, especially in developing countries [9]. These infectious diseases can be successfully treated with specific antimicrobial interventions, and thus careful and extensive systemic work up of these causes must be performed to rule out infections prior to the institution of treatment [10].

\section{Juvenile idiopathic arthritis}

Juvenile idiopathic arthritis is a systemic autoimmune disease and can be defined as a group of idiopathic arthritis diseases that occurs before the age of 16 and persists for at least 6 weeks. The peak age of onset is between the ages of 6 months and 4 years. Juvenile idiopathic arthritis affects approximately 70,000 children in the US. Its pathogenesis appears to be multifactorial; genetic predisposition has been implicated, and the onset is possibly triggered by microbial contact [6]. The most common extra-articular manifestation of juvenile idiopathic arthritis is intraocular inflammation. The prevalence of uveitis in juvenile idiopathic arthritis patients can range from $4 \%$ to $38 \%$ and varies by juvenile idiopathic arthritis subtype, the most common being oligoarticular onset [11,12]. Juvenile idiopathic arthritis patients with uveitis typically present with bilateral non-granulomatous uveitis. However, granulomatous uveitis with mutton-fat keratic precipitates can occur [13]. Rarely, the posterior segment of the eye can also be involved.
The leading cause of visual impairment in juvenile idiopathic arthritis is complicated cataract and band keratopathy. Other complications associated are secondary glaucoma, epiretinal membrane formation, macular hole, hypotony with ciliary body shutdown or atrophy. The diagnosis should include a careful review of the symptoms and general examination of patients, often with rheumatologist co-management. Early diagnosis and prompt treatment to achieve complete resolution of inflammation is the goal for the prevention of sight-threatening complications in juvenile idiopathic arthritis $[6,14,15]$.

\section{Pars planitis}

The term "intermediate uveitis" should be used for that subset of uveitis where the vitreous is the major site of the inflammation. It is estimated that intermediate uveitis represents one-fifth of all cases of pediatric uveitis [6]. According to Standardization of Uveitis Nomenclature (SUN) criteria, the term "pars planitis" should be used only when there is intermediate uveitis with snowbank or snowball formation, in the absence of an associated infection or systemic disease [16]. Identification of vitreous cells at slit lamp examination is the sine qua non of this disease. Usually, patients with pars planitis present with complaints of blurry vision and floaters. Symptoms such as redness, painful eyes and tearing are less common. Children can be diagnosed with intermediate uveitis incidentally on routine ophthalmic examination because significant anterior segment inflammation is not very frequent. Children with pars planitis have worse visual acuity, both at diagnosis and at follow-up, than adults. Complications such as cataract, cystoid macular edema, secondary glaucoma, vitreous hemorrhage and retinal detachment can occur $[4,17]$.

\section{Treatment}

\section{The stepladder approach}

The preferred treatment strategy for non-infectious uveitis is the so-called "stepladder approach". With anterior inflammation, aggressive topical corticosteroids are initiated as the first-line treatment; if this does not quiet the inflammation, local corticosteroid injections are added to the regimen. Careful monitoring of intraocular pressure during a topical steroid regimen is important. Systemic steroid treatment can also be implemented, but in brief courses (less than three months), as there can be serious long-term side effects, including growth retardation due to adrenal suppression and premature closure of the epiphyseal plates [18]. Weight gain, hyperglycemia, infection, or osteoporosis can also occur due to extensive use of corticosteroid [18]. As topical steroids are generally ineffective at penetrating the eyeball to the posterior segment, systemic treatment or regional injections may be 
Table I. Differential diagnosis of pediatric uveitis

\begin{tabular}{ll}
\hline Anterior non-granulomatous & Anterior granulomatous \\
\hline - Juvenile idiopathic arthritis & - Sarcoidosis \\
- Herpetic disease & • Herpetic disease \\
- HLA-B27-associated disease & - Inflammatory bowel disease \\
- Systemic lupus erythematosus & - Syphilis \\
- Fuchs' heterochromic iridocyclitis & - Lyme disease \\
- Kawasaki disease & - Leprosy \\
- Tubulointerstitial nephritis and uveitis & - Tuberculosis \\
- Trauma & - Trauma \\
- Viral syndromes & - Sympathetic ophthalmia \\
- Post bacterial infection (Yersinia, Streptococcal) & - Fungal disease \\
- Amantiades-Behçet's disease & - Multiple sclerosis \\
- Human immunodeficiency virus & \\
- Leukemia & \\
- Drug induced & \\
- CINCA/NOMID & \\
- Juvenile xanthogranuloma
\end{tabular}

\begin{tabular}{|c|c|}
\hline Intermediate uveitis & Panuveitis \\
\hline - Pars planitis & - Amantiades-Behçet's disease \\
\hline - Juvenile idiopathic arthritis & - Sympathetic ophthalmia \\
\hline - Multiple sclerosis & - Orbital pseudotumor \\
\hline - Lyme disease & - Vogt-Koyanagi-Harada syndrome \\
\hline - Cat-scratch disease & - Lyme disease \\
\hline - Toxocariasis & - Cat-scratch disease \\
\hline - Masquerades & - Masquerades \\
\hline - Sarcoidosis & - Sarcoidosis \\
\hline - Tubulointerstitial nephritis and uveitis & - Tubulointerstitial nephritis and uveitis \\
\hline \multicolumn{2}{|l|}{ - Syphilis } \\
\hline \multicolumn{2}{|l|}{$\begin{array}{l}\text { - Familial juvenile systemic granulomatosis } \\
\text { (Blau syndrome) }\end{array}$} \\
\hline Posterior uveitis without vasculitis & Posterior uveitis with vasculitis \\
\hline - Toxocariasis & - Toxoplasmosis \\
\hline • Leukemia & - Sarcoidosis \\
\hline - Tuberculosis & • Syphilis \\
\hline - Vogt-Koyanagi-Harada syndrome & - Lyme disease \\
\hline - Trauma (intraocular foreign body) & - Acute retinal necrosis (HSV/VZV) \\
\hline - Rubella & - Cytomegalovirus retinitis \\
\hline - Diffuse subacute unilateral neuroretinitis & - Human immunodeficiency virus \\
\hline • Endophthalmitis & • Multiple sclerosis \\
\hline - Presumed ocular histoplasmosis syndrome & - Paraviral syndrome \\
\hline - Masquerades & - Masquerades \\
\hline \multirow[t]{6}{*}{ - White dot syndromes } & - Kawasaki disease \\
\hline & - Amantiades-Behçet's disease \\
\hline & - Polyarteritis nodosa \\
\hline & - Granulomatosis with polyangiitis (Wegener's) \\
\hline & - Systemic lupus erythematosus \\
\hline & •Inflammatory bowel disease \\
\hline
\end{tabular}

HLA, human leukocyte antigen; HSV, herpes simplex viruses; NOMID, Neonatal-onset multisystem inflammatory disease; CINCA, Chronic infantile neurological, cutaneous and articular syndrome; VZV, Varicella zoster virus. Adapted with permission from Diagnosis and Treatment of Uveitis, Chapter 8I [6]. 
Table 2. Differential diagnosis of intermediate and posterior pediatric uveitis: age at presentation

\begin{tabular}{ll}
\hline Infants (age 0-2 years) & Children (age 2-10 years) \\
\hline - Toxoplasmosis & - Toxoplasmosis \\
- Lymphocytic choriomeningitis & - Toxocariasis \\
virus & - Leukemia \\
- HSV retinitis & - Juvenile idiopathic arthritis \\
- Retinoblastoma & - Cat-scratch disease \\
- Rubella & - Subacute sclerosing panencephalitis \\
- Congenital syphilis & - CINCA/NOMID syndrome \\
& - Familial juvenile systemic \\
& granulomatosis (Blau syndrome) \\
\hline Adolescents (10-20 years) & Any childhood age \\
\hline - Juvenile idiopathic arthritis & - HIV retinopathy \\
- Pars planitis & - CMV retinitis \\
- HLA-B27 associated disease & - Acute retinal necrosis \\
- Toxoplasmosis & - Endophthalmitis \\
- Acute posterior multifocal placoid & - Sarcoidosis \\
pigment epitheliopathy & - Lyme disease \\
- Presumed ocular histoplasmosis & - Cat-scratch disease \\
syndrome & - Tubulointerstitial nephritis and \\
- Diffuse subacute unilateral & uveitis syndrome \\
neuroretinitis & - Tuberculosis \\
- Leukemia & - Vogt-Koyanagi-Harada syndrome \\
& - Sympathetic ophthalmia \\
& - Amantiades-Behçet's disease \\
& body) \\
\hline
\end{tabular}

HLA, human leukocyte antigen; NOMID, Neonatal-onset multisystem inflammatory disease; CINCA, Chronic infantile neurological, cutaneous and articular syndrome. Adapted with permission from Diagnosis and Treatment of Uveitis, Chapter 8I [6].

the first-line therapy in cases of intermediate or posterior uveitis.

When tapering the corticosteroids leads to a recurrence of inflammation, the second step in the ladder involves systemic non-steroidal anti-inflammatory medications (NSAIDs). Naproxen and tolmentin have the longest history of successful use among pediatric rheumatologists [19]. Celecoxib and diflunisal, in our experience, proved to be useful in controlling human leukocyte antigen (HLA)B27-associated anterior uveitis such as that seen in ankylosing spondylitis and the other spondyloarthropathies [20]. However, should these therapies prove to be insufficient, early initiation of immunomodulatory therapy is key. Methotrexate is generally the first choice, as it has a well-established safety and efficacy profile in children and does not appear to increase the risk of cancer [21]. We typically begin treatment of a child at $0.15 \mathrm{mg} / \mathrm{kg}$ orally once a week, and increase the dosage every 6-8 weeks until stable quiescence, entirely without corticosteroids, is observed. In juvenile idiopathic arthritis-associated uveitis, the dose required to keep uveitis in remission is typically higher than that required to relieve joint symptoms, but children generally tolerate higher doses well [19]. Additionally, dosage of methotrexate is based on weight, so dose adjustments must be made carefully as the child grows. If uveitis persists after several dose increases, it is advisable to switch to the subcutaneous dosage form, as the bioavailability after oral administration can be variable. If the dose exceeds $17.5 \mathrm{mg}$, we typically switch to the subcutaneous form. Significant potential side effects of methotrexate include bone marrow suppression, hepatotoxicity, and interstitial pneumonitis, which is why it is crucial that a provider must be an expert in the prescribing and monitoring of methotrexate therapy, or work with a rheumatologist. As methotrexate is a folic acid analogue, adding a regimen of $1 \mathrm{mg}$ of folic acid every day of the week except the day that the medication is taken, or $5 \mathrm{mg}$ of leukovorin once a week, reduces the risk of side effects.

Approximately three-quarters of juvenile idiopathic arthritis patients experience improvement in inflammation with methotrexate as monotherapy [21]. In those that do not improve, the other classic immunomodulators should be explored: azathioprine, mycophenolate mofetil, or cyclosporine can be substituted. Azathioprine is moderately effective in both children and adults [22], but is less commonly prescribed in children, as gastrointestinal side effects are more common than in the other antimetabolites [23]. Mycophenolate mofetil may be effective in quieting inflammation in over half of those cases refractory to methotrexate [24] and, in non-juvenile idiopathic arthritis uveitis, is sometimes used instead of methotrexate as a firstline corticosteroid-sparing agent [8].

Cyclosporine A (CsA), a calcineurin inhibitor that prevents T-cell activation, can be used on its own or in conjunction with the former two antimetabolites. Cyclosporine has been observed to be particularly effective in patients with Behçet's disease-associated uveitis [25]; in children with juvenile idiopathic arthritis-associated uveitis, cyclosporine is more effective when used in combination with methotrexate [26]. Initial dosing is recommended at 3-5 mg/ $\mathrm{kg} /$ day divided into two doses, followed by a maintenance dose of $2-3 \mathrm{mg} / \mathrm{kg} /$ day $[6,27]$. Less widely used T-cell inhibitors include tacrolimus and sirolimus and, although current data in children are lacking, with more research they may become options in the future. Some individuals may experience hypertension as a side effect of both cyclosporine and tacrolimus, so monitoring of blood pressure is warranted. Additionally, these drugs may have 
nephrotoxic effects, and thus should not be given in tandem [27].

\section{Biologic response modifiers}

The most recent additions to the treatment options of both children and adults with uveitis are the biologic therapies, which are typically monoclonal antibodies designed to halt the inflammatory cascade by binding and inhibiting proinflammatory cytokines.

The tumor necrosis factor (TNF)-alpha inhibitors infliximab and adalimumab are shown to be the most effective in their class at controlling ocular inflammation, and can be used on their own or in combination with classic immunomodulatory therapy, when one or the other is insufficient [28-31]. An expert panel of the American Uveitis Society released its recommendations on the use of infliximab and adalimumab as first-line agents in the treatment of Behçet's, as second-line in the treatment of juvenile idiopathic arthritis-associated uveitis after methotrexate, and as potential second-line agents for posterior or panuveitis, or in patients who have failed to respond to the classic immunomodulators [32]. Infliximab, a mouse/human chimeric antibody, is given by intravenous infusion at doses of $5-20 \mathrm{mg} / \mathrm{kg}$ every 4 weeks, after an induction period [28]. Low dose antimetabolite therapy is usually given in conjunction with infliximab to prevent antichimeric antibodies [33]. Adalimumab, a fully humanized monoclonal antibody, may be given at a dose of 20-40 mg every 7 to 14 days and is administered by subcutaneous injection. While infliximab has a faster onset, adalimumab has less risk of infection and greater ease of administration by subcutaneous injection. Some data suggest that adalimumab may be slightly more effective than infliximab in achieving remission $[29,34]$, while other studies indicate no difference between the two. Trials are currently underway to evaluate the effectiveness of methotrexate and adalimumab combined therapy versus adalimumab monotherapy [35].

Golimumab and certolizumab pegol are two other drugs in this class that have been used to treat other autoimmune diseases such as rheumatoid arthritis. As for their effectiveness in uveitis, data are limited to case reports [36,37]. Etanercept, although effective in treating the systemic rheumatological manifestations of juvenile idiopathic arthritis, should not be used in preference to the other TNF-alpha inhibitors, as it is less effective in controlling ocular inflammation [32].

Biologics that target other immune cells may aid in the cases of uveitis that are refractory to anti-TNF therapies but the current data, especially in children, are limited.
Tocilizumab, an inhibitor of the proinflammatory cytokine interleukin (IL)-6, has been noted in several case reports to be potentially effective in the treatment of uveitis that does not respond to anti-TNF agents $[38,39]$. Rituximab, an anti-CD20 monoclonal antibody approved for treatment of rheumatoid arthritis, lymphomas, and leukemias, was shown to control uveitis in 7 out of 10 patients with juvenile idiopathic arthritis uncontrolled by anti-TNF or immunomodulatory agents [40]. Case reports also show that abatacept, a cytotoxic T-lymphocyte antigen (CTLA)-4 fusion protein that inhibits T-cell co-stimulation, is another agent that has been successful in controlling inflammation in refractory juvenile idiopathic arthritis-associated uveitis [41,42]. Many clinical trials are underway to assess potential biologic therapies, including sarilumab (anti-IL6), anakinra (anti-IL1), ustekinumab (anti-IL23, anti-IL12), canakinumab (anti-IL-13), and gevokizumab (anti-IL-1 $\beta$ ).

Intravenous immunoglobulin represents another option for uveitis refractory to other therapies; it is unique in that it treats autoimmune disease without suppressing the immune system. Intravenous immunoglobulin is currently used in rheumatology and ophthalmology for vasculitis, systemic lupus erythematosus (SLE), mucous membrane pemphigoid and uveitis, and is currently being studied in a wide range of other conditions [43]. Case reports show intravenous immunoglobulin as effective in treating uveitis refractory to other treatments, although more data are necessary $[44,45]$. Doses for immunomodulation are given at 2 grams/kg by intravenous infusion over a course of 2-3 days in 1 week, once monthly.

\section{Other treatments}

The cytotoxic alkylating agents chlorambucil and cyclophosphamide are almost always effective in reducing inflammation, but are used only as a last-resort due to potential serious long-term side effects [46,47]. Prolonged use increases the risk of developing a malignancy. This risk can be mitigated by limiting the total cumulative dose and duration to one year or less. Alkylating agents are sometimes associated with significant gonadal dysfunction or infertility in post-pubescent patients, and the effects may or may not be reversible. In girls, ovarian suppression can be induced to protect ova during the course of treatment [48].

Procedural interventions, such as pars plana vitrectomy, are preferred to systemic treatments in certain cases of recurrent pars planitis in order to mechanically clear out inflammatory cells. In our retrospective medical record review of 20 pediatric patients (28 eyes) who underwent pars plana vitrectomy, quiescence of uveitis was achieved at last follow-up, with or without adjuvant therapy, in 
27 of 28 eyes (96\%). Mean follow-up time after surgery was 13.5 months [49]. Providers should discuss both surgical interventions and long-term immunomodulatory therapy with the family to decide on the most appropriate option.

Implantable corticosteroid-eluting intravitreal devices, such as the fluocinolone acetonide implant (Retisert ${ }^{\circ}$ ) or the dexamethasone implant (Ozurdex ${ }^{\circ}$ ), represent surgical options for the recalcitrant case. However, the data in children are quite lacking. Retisert ${ }^{\circledR}$ releases $0.59 \mathrm{mg}$ of fluocinolone acetonide over 30 months [50] and Ozurdex ${ }^{\circ}$ releases $0.7 \mathrm{mg}$ of dexamethasone over 6 months [51]. Some case reports have indicated successful treatment of intermediate uveitis or panuveitis with the dexamethasone implant with minimal side effects at 3 [52] or 6 [53] months. However, with both devices, there remains a substantial risk of developing cataract and glaucoma, though the risk may be higher with the fluocinolone acetonide implant [54]. In the interest of avoiding future cataract surgery and other complications, these devices are used infrequently in children.

\section{Conclusion}

The goal with any patient is ultimately steroid-free durable remission, in total quiescence for a minimum of two years before tapering therapies. Pediatric uveitis represents a unique challenge in both diagnosis and therapy, but it is essential that appropriate treatment be instated promptly. An ophthalmologist should still monitor children with juvenile idiopathic arthritis but without ocular symptoms so that treatment may be instated rapidly in the event that uveitis develops. The stepladder approach is the preferable method, beginning with corticosteroid therapy to quickly abolish inflammation, and then advancing to classic immunomodulators, adding biological therapies if necessary, in pursuit of steroid-free remission. Ophthalmologists not comfortable with prescribing and monitoring immunomodulatory or biologic therapies should not delay in collaborating with a rheumatologist familiar with the administration of these drugs, or referring patients to another specialist, as the long-term consequences of prolonged corticosteroid therapy are undesirable at best, not to mention the vision-threatening outcomes of recurrent inflammation.

\section{Abbreviations}

HLA, human leukocyte antigen; IL, interleukin; TNF, tumor necrosis factor.

\section{Disclosures}

The authors declare that they have no disclosures.

\section{References}

I. Gritz DC, Wong IG: Incidence and prevalence of uveitis in Northern California; the Northern California Epidemiology of Uveitis Study. Ophthalmology 2004, I I I:49 I-500; discussion 500.

2. Acharya NR, Tham VM, Esterberg E, Borkar DS, Parker JV, Vinoya $A C$, Uchida $A$ : Incidence and prevalence of uveitis: results from the Pacific Ocular Inflammation Study. JAMA Ophthalmol 2013, I3 I: I405-12.

3. Smith JA, Mackensen F, Sen HN, Leigh JF, Watkins AS, Pyatetsky D, Tessler HH, Nussenblatt RB, Rosenbaum JT, Reed GF, Vitale S, Smith JR, Goldstein DA: Epidemiology and Course of Disease in Childhood Uveitis. Ophthalmology 2009, I I 6: I544-5 I.el.

4. Malinowski SM, Pulido JS, Folk JC: Long-term visual outcome and complications associated with pars planitis. Ophthalmology 1993 , 100:818-24; discussion 825.

5. Rosenberg KD, Feuer WJ, Davis JL: Ocular complications of pediatric uveitis. Ophthalmology 2004, I I I:2299-306.

6. Foster CS, Vitale Albert T, Kump L: Pediatric Uveitis. In Diagnosis and treatment of uveitis. 2nd edition. New Delhi; London: Jaypee Brothers Medical; 2013: I214-53.

7. Gregory AC 2nd, Kempen JH, Daniel E, Kaçmaz RO, Foster CS, Jabs DA, Levy-Clarke GA, Nussenblatt RB, Rosenbaum JT, Suhler EB, Thorne JE; Systemic Immunosuppressive Therapy for Eye Diseases Cohort Study Research Group: Risk factors for loss of visual acuity among patients with uveitis associated with juvenile idiopathic arthritis: the Systemic Immunosuppressive

Therapy for Eye Diseases Study. Ophthalmology 2013, 120:186-92.

FlOOOPrime

RECOMMENDED

8. Mehta PJ, Alexander JL, Sen HN: Pediatric uveitis: new and future treatments. Curr Opin Ophthalmol 2013, 24:453-62.

FlOOOPrime

9. Mandelcorn ED: Infectious causes of posterior uveitis. Can J Ophthalmol J Can Ophtalmol 2013, 48:31-9.

FlOOOPrime RECOMMENDED

10. Madigan WP, Raymond WR, Wroblewski KJ, Thebpatiphat N, Birdsong RH, Jaafar MS: A Review of Pediatric Uveitis: Part I. Infectious Causes and the Masquerade Syndromes. J Pediatr Ophthalmol Strabismus 2008, 45: I40-9.

II. Chalom EC, Goldsmith DP, Koehler MA, Bittar B, Rose CD, Ostrov BE, Keenan GF: Prevalence and outcome of uveitis in a regional cohort of patients with juvenile rheumatoid arthritis. J Rheumatol 1997, 24:203।-4.

FlOOOPrime

RECOMMENDED

12. Kotaniemi K, Kautiainen H, Karma A, Aho K: Occurrence of uveitis in recently diagnosed juvenile chronic arthritis: a prospective study. Ophthalmology 200I, I 08:207I-5.

FlOOOPrime

RECOMMENDED

13. Keenan JD, Tessler HH, Goldstein DA: Granulomatous inflammation in juvenile idiopathic arthritis-associated uveitis. J AAPOS 2008, I 2:546-50.

FlOOOPrime RECOMMENDED

14. Nguyen QD, Foster CS: Saving the vision of children with juvenile rheumatoid arthritis-associated uveitis. JAMA I998, 280: I |33-4.

15. Majumder PD, Biswas J: Pediatric uveitis: An update. Oman J Ophthalmol 2013, 6:140-50.

16. Jabs DA, Nussenblatt RB, Rosenbaum JT; Standardization of Uveitis Nomenclature (SUN) Working Group: Standardization of uveitis 
nomenclature for reporting clinical data. Results of the First International Workshop. Am J Ophthalmol 2005, I 40:509-I6.

17. Guest S, Funkhouser E, Lightman S: Pars planitis: a comparison of childhood onset and adult onset disease. Clin Experiment Ophthalmol 200I, 29:8I-4.

\section{FlOOOPrime}

\section{RECOMMENDED}

18. Buchman AL: Side effects of corticosteroid therapy. J Clin Gastroenterol 200 I, 33:289-94.

19. Vitale A, Kump LI, Foster CS: Uveitis affecting infants and children. In Pediatric retina. 2nd edition. Philadelphia: Wolters Kluwer Health/Lippincott Williams \& Wilkins; 2013

20. Fiorelli VMB, Bhat P, Foster CS: Nonsteroidal anti-inflammatory therapy and recurrent acute anterior uveitis. Ocul Immunol Inflamm 2010, 18:1 I6-20.

21. Simonini G, Paudyal P, Jones GT, Cimaz R, Macfarlane G]: Current evidence of methotrexate efficacy in childhood chronic uveitis: a systematic review and meta-analysis approach. Rheumatol Oxf Engl 2013, 52:825-31

\section{FlOOPrime} RECOMMENDED

22. Pasadhika S, Kempen $\mathrm{H}$, Newcomb CW, Liesegang TL, Pujari SS, Rosenbaum JT, Thorne JE, Foster CS, Jabs DA, Levy-Clarke GA, Nussenblatt RB, Suhler EB: Azathioprine for ocular inflammatory diseases. Am J Ophthalmol 2009, I48:500-9.e2.

23. Goebel JC, Roesel M, Heinz C, Michels H, Ganser G, Heiligenhaus A: Azathioprine as a treatment option for uveitis in patients with juvenile idiopathic arthritis. $\mathrm{Br} J$ Ophthalmol 20II, 95:209-13.

24. Sobrin L, Christen W, Foster CS: Mycophenolate mofetil after methotrexate failure or intolerance in the treatment of scleritis and uveitis. Ophthalmology 2008, I I5:|4|6-2I.el.

25. Ozdal PC, Ortaç S, Taskintuna I, Firat E: Long-term therapy with low dose cyclosporin A in ocular Behçet's disease. Doc Ophthalmol Adv Ophthalmol 2002, I05:301-12.

\section{FlOOOPrime}

26. Tappeiner C, Roesel M, Heinz C, Michels H, Ganser G, Heiligenhaus A: Limited value of cyclosporine $A$ for the treatment of patients with uveitis associated with juvenile idiopathic arthritis. Eye Lond Engl 2009, 23: I I 92-8.

\section{FlOOOPrime}

\section{RECOMMENDED}

27. Gallego-Pinazo R, Dolz-Marco R, Martínez-Castillo S, Arévalo JF, DíazLlopis $M$ : Update on the principles and novel local and systemic therapies for the treatment of non-infectious uveitis. Inflamm Allergy Drug Targets 2013, I 2:38-45.

28. Tambralli A, Beukelman T, Weiser P, Atkinson TP, Cron RQ, Stoll ML: High doses of infliximab in the management of juvenile idiopathic arthritis. J Rheumatol 2013, 40:1749-55.

29. Zannin ME, Birolo C, Gerloni VM, Miserocchi E, Pontikaki I, Paroli MP, Bracaglia C, Shardlow A, Parentin F, Cimaz R, Simonini G, Falcini F, Corona F, Viola S, De Marco R, Breda L, La Torre F, Vittadello F, Martini G, Zulian F: Safety and efficacy of infliximab and adalimumab for refractory uveitis in juvenile idiopathic arthritis: I-year followup data from the Italian Registry. I Rheumatol 20I3, 40:74-9.

\section{FlOOOPrime} RECOMMENDED

30. Ardoin SP, Kredich D, Rabinovich E, Schanberg LE, Jaffe GJ: Infliximab to treat chronic noninfectious uveitis in children: retrospective case series with long-term follow-up. Am J Ophthalmol 2007, I 44:844-9.

\section{FlOOOPrime}

\section{RECOMMENDED}

31. Doycheva D, Zierhut M, Blumenstock G, Stuebiger N, Januschowski K, Voykov B, Deuter C: Immunomodulatory therapy with tumour necrosis factor $\alpha$ inhibitors in children with antinuclear antibody-associated chronic anterior uveitis: long-term results. $\mathrm{BrJ}$ Ophthalmol 2014, 98:523-8.

\section{FlOOOPrime \\ RECOMMENDED}

32. Levy-Clarke G, Jabs DA, Read RW, Rosenbaum JT, Vitale A, Van Gelder RN: Expert Panel Recommendations for the Use of AntiTumor Necrosis Factor Biologic Agents in Patients with Ocular Inflammatory Disorders. Ophthalmology 2013, I 2 I:785-96.e3.

\section{FlOOOPrime
RECOMMENDED}

33. Horneff G: Update on biologicals for treatment of juvenile idiopathic arthritis. Expert Opin Biol Ther 2013, I3:36I-76.

34. Simonini G, Taddio A, Cattalini M, Caputo R, De Libero C, Naviglio S, Bresci C, Lorusso M, Lepore L, Cimaz R: Prevention of flare recurrences in childhood-refractory chronic uveitis: an openlabel comparative study of adalimumab versus infliximab. Arthritis Care Res 2011, 63:612-8.

35. Ramanan AV, Dick AD, Benton D, Compeyrot-Lacassagne S, Dawoud D, Hardwick B, Hickey $H$, Hughes D, Jones A, Woo P, Edelsten C, Beresford MW; SYCAMORE Trial Management Group: A randomised controlled trial of the clinical effectiveness, safety and costeffectiveness of adalimumab in combination with methotrexate for the treatment of juvenile idiopathic arthritis associated uveitis (SYCAMORE Trial). Trials 2014, I5:I4.

36. Miserocchi E, Modorati G, Pontikaki I, Meroni PL, Gerloni V: Longterm Treatment with Golimumab for Severe Uveitis. Ocul Immunol Inflamm 2014, 22:90-5.

\section{FlOOOPrime}

37. Sánchez-Cano D, Callejas-Rubio JL, Ruiz-Villaverde R, Ríos-Fernández R, Ortego-Centeno N: Off-label uses of anti-TNF therapy in three frequent disorders: Behçet's disease, sarcoidosis, and noninfectious uveitis. Mediators Inflamm 2013, 20 I3:286857.

38. Tappeiner C, Heinz C, Ganser G, Heiligenhaus A: Is tocilizumab an effective option for treatment of refractory uveitis associated with juvenile idiopathic arthritis? J Rheumatol 20I2, 39:I294-5.

39. Muselier A, Bielefeld P, Bidot S, Vinit J, Besancenot J-F, Bron A: Efficacy of tocilizumab in two patients with anti-TNF-alpha refractory uveitis. Ocul Immunol Inflamm 20I I, I 9:382-3.

40. Heiligenhaus A, Miserocchi E, Heinz C, Gerloni V, Kotaniemi K: Treatment of severe uveitis associated with juvenile idiopathic arthritis with anti-CD20 monoclonal antibody (rituximab). Rheumatol Oxf Engl 201 I, 50:1390-4.

\section{FlOOOPrime}

4I. Kenawy N, Cleary G, Mewar D, Beare N, Chandna A, Pearce I: Abatacept: a potential therapy in refractory cases of juvenile idiopathic arthritis-associated uveitis. Graefes Arch Clin Exp Ophthal$\mathrm{mol} 201 \mathrm{I}, \mathbf{2 4 9 : 2 9 7 - 3 0 0 .}$

42. Zulian F, Balzarin M, Falcini F, Martini G, Alessio M, Cimaz R, Cimino L, Zannin ME: Abatacept for severe anti-tumor necrosis factor alpha refractory juvenile idiopathic arthritis-related uveitis. Arthritis Care Res 2010, 62:821-5.

\section{FlOOOPrime \\ RECOMMENDED}

43. Jolles S, Sewell WAC, Misbah SA: Clinical uses of intravenous immunoglobulin. Clin Exp Immunol 2005, I42: I- II.

44. LeHoang P, Cassoux N, George F, Kullmann N, Kazatchkine MD: Intravenous immunoglobulin (IVIg) for the treatment of birdshot retinochoroidopathy. Ocul Immunol Inflamm 2000, 8:49-57.

45. Rosenbaum JT, George RK, Gordon C: The treatment of refractory uveitis with intravenous immunoglobulin. Am J Ophthalmol I999, I 27:545-9.

46. Pujari SS, Kempen JH, Newcomb CW, Gangaputra S, Daniel E, Suhler EB, Thorne JE, Jabs DA, Levy-Clarke GA, Nussenblatt RB, Rosenbaum JT, 
Foster CS: Cyclophosphamide for ocular inflammatory diseases. Ophthalmology 2010, II 7:356-65.

47. Larson $T$, Nussenblatt RB, Sen HN: Emerging drugs for uveitis. Expert Opin Emerg Drugs 201I, 16:309-22.

48. Foster CS, Vitale AT: Immunosuppressive chemotherapy. In Diagnosis and Treatment of Uveitis. 2nd edition. New Delhi; London: Jaypee Brothers Medical; 2013: 238-93.

49. Giuliari GP, Chang PY, Thakuria P, Hinkle DM, Foster CS: Pars plana vitrectomy in the management of paediatric uveitis: the Massachusetts Eye Research and Surgery Institution experience. Eye Lond Engl 2010, 24:7-13.

50. Retisert [package insert]. Rochester, NY: Bausch \& Lomb Inc. 2012.

51. Ozurdex [package insert]. Irvine, CA: Allergan Inc. 2013.
52. Bourgault S, Aroichane M, Wittenberg LA, Lavallée A, Ma PE: Treatment of refractory uveitic macular edema with dexamethasone intravitreal implants in a pediatric patient with bilateral granulomatous idiopathic panuveitis: a case report. J Ophthalmic Inflamm Infect 2013, 3:61.

53. Taylor SRJ, Tomkins-Netzer O, Joshi L, Morarji J, McLoone E, Lightman S: Dexamethasone implant in pediatric uveitis. Ophthalmology 20I2, II 9:24I2-24I2.e2.

\section{FlOOPrime}

RECOMMENDED

54. Patel CC, Mandava N, Oliver SCN, Braverman R, Quiroz-Mercado H, Olson JL: Treatment of intractable posterior uveitis in pediatric patients with the fluocinolone acetonide intravitreal implant (Retisert). Retina Phila Pa 2012, 32:537-42. 